\title{
Thermoelectrically Based Approaches to Reduce Adhesive Wear During Blanking
}

\author{
MARKUS WELM $\odot{ }^{1,3}$ PHILIPP TRÖBER, ${ }^{1}$ HANNES ALOIS WEISS, ${ }^{1}$ \\ PETER DEMMEL, ${ }^{2}$ ROLAND GOLLE ${ }^{1}$ and WOLFRAM VOLK ${ }^{1}$
}

1.-Chair of Metal Forming and Casting, Technical University of Munich, 85748 Garching, Germany. 2.-MAN Truck \& Bus AG, 80995 Munich, Germany. 3.-e-mail: markus.welm@utg.de

\begin{abstract}
Almost every metal mass product goes through a blanking process. Especially when processing aluminum, adhesive wear is the main determinant of cost efficiency. Many investigations on wear-influencing factors have been conducted so far, but one major determinant is almost unnoticed, thermoelectric phenomena. Due to the Seebeck effect, thermoelectricity arises in every blanking tool. Recently published investigations show that the combination of tool and workpiece materials has a strong influence on occurring thermoelectric currents and thus on adhesive wear development. This can be traced back to dependence of the current strength and direction on the materialspecific Seebeck coefficient. This article addresses the same phenomenon for a new parameter spectrum. Blanking experiments with aluminum EN AW 5083 were performed, investigating both thermoelectric currents and the amount of adhesive wear. Furthermore, the impact of external currents influencing the naturally occurring thermoelectricity on wear is shown. Improved measurements with a laser confocal microscope reveal a close correlation between the thermoelectric current profiles and adhesive wear pattern on the lateral surface of the punch. Together with a variation of tool material among highspeed steel 1.3343, stainless steel 1.4301 and cemented carbide CF-H40S, a strong relation between the Seebeck coefficients, electrical currents and tool wear could be found. Therefore, the actual findings confirm, deepen and extend previous results concerning thermoelectricity and adhesive wear.
\end{abstract}

\section{INTRODUCTION}

Blanking and cold forming are two of the most common manufacturing processes for automotive parts, household appliances, electronic components and nearly every large-scale metal production good. Increased environmental demands on cars and thus fuel-efficiency claims require special attention to lightweight architecture. For this purpose, aluminum is indispensable. ${ }^{i}$ Furthermore, aluminum is of increased importance respecting visible parts in domestic appliances and as a substitute material for replacing cost-intensive copper in electronic applications. One big challenge in processing aluminum is its high tendency to adhesive tool wear formation, resulting from interactions with the tool material on a micro- and nanoscopic level. ${ }^{2}$
Adhesive wear primarily arises in areas with local stress peaks and consequently high temperatures. ${ }^{3}$ This takes place during cold metal forming, e.g., at roughness asperities in contact. The formation of adhesive wear is very temperature sensitive. Groche and Nitzsche showed that an increase of just $15^{\circ} \mathrm{C}$ in a strip drawing test results in a significant increase of adhesive wear quantity. ${ }^{4}$ Adhesive wear entails negative effects like part quality reduction, especially at the cutting surface. Its removal forms tinsel, ${ }^{5}$ which dents into the sheet metal and raises the number of defective parts. ${ }^{6}$ Furthermore, those metal fragments cause short circuits in the electrical components and lead to unexpected machine downtime. To meet part quality, cycle time and tool service live requirements, it is essential to reduce the adhesive wear. ${ }^{7}$ State-ofthe-art prevention strategies are tool coatings and lubrication. Both strategies raise investment costs, 
and lubricant additives in particular are often harmful to the environment. ${ }^{8}$ Furthermore, their removal before subsequent production steps, e.g., welding operations, is necessary and causes higher cycle times and further costs.

Despite their high degree of importance, the development mechanisms of adhesive wear are insufficiently understood. Czichos and Habig describe the process as follows: The passivating oxide layer cracks because of plastic deformation; consequently, an adhesive bonding of sheet metal and tool material occurs. ${ }^{9}$ Further relative movement of the punch separates the cohesive bonding in the sheet metal, and a material transfer to the punch occurs. Besides temperature, thermoelectricity is a significant influencing factor. Due to the Seebeck effect, this phenomenon appears in every electrical circuit that is formed by two different conductors that are connected and subjected to a temperature gradient. ${ }^{10}$ In an open electric circuit, a temperature-proportional voltage emerges that depends on the difference between the thermoelectric material properties of the conductors, represented by the Seebeck coefficient. In an electric short circuit configuration, the thermoelectric voltage generates circular currents. These currents also depend on the electrical resistance.

Differing chemical compositions of sheet and tool material result in differing thermoelectric properties. Both materials form such a circuit. Consequently, thermoelectric currents occur during every sheet metal manufacturing process because of a temperature increase in the forming zone. Three effects cause this temperature increase: One is the dissipation of up to $95 \%$ of the plastic deformation work into heat, ${ }^{11,12}$ with only a minor amount stored in dislocations and other lattice defects. ${ }^{13}$ Another reason is macroscopic friction, and the last one is microscopic friction on the atomic level. ${ }^{14}$ Maximum occurring contact temperatures are significantly dependent on process parameters such as die clearance, cutting edge radii and punch speed as well as wear. Whereas the first two issues affect the spatial expansion of the forming zone, the area in which the plastic work dissipates, punch speed influences the available time for heat transfer effects. ${ }^{15}$

Awakow and Ritschkin investigated the influence of thermoelectricity in drilling and found strong correlations among tool lifetime, current direction and strength as well as the combination of tool and workpiece material. ${ }^{16}$ Opitz measured very high thermoelectric currents of up to $5 \mathrm{~A}$ during steel turning. ${ }^{17}$ An extension of tool lifetime by electrically insulating the active elements and consequently interrupting thermoelectric currents was Bobrovskij's research field. ${ }^{18}$ Ellis and Barrow deepened the knowledge and showed that whether insulation has a positive or negative effect on adhesive wear depends on the internal resistance of the machine. ${ }^{19}$ Shan expanded upon the latter by considering thermoelectric currents generated in different parts of the machine ${ }^{20}$ Determinants of thermoelectric current development and its manipulation using external currents were examined by Uehara. ${ }^{21}$ Tröber et al. investigated the influence of Seebeck coefficients and thermoelectric currents on adhesive tool wear during blanking and were also able to show strong correlations. ${ }^{22,23}$

This article builds upon previously published material and addresses the influences of thermoelectricity on adhesive wear during blanking. For this purpose, a new parameter spectrum and experimental setup as well as a reproducible punch speed profile were used. Together with the previously unexamined high-speed steel 1.3343 having a similar Seebeck coefficient to the previously investigated cold working steel 1.2379 and the investigation of adhesive wear with a laser confocal microscope, it was possible to confirm and extend the previous results. Beside the influence of thermoelectric currents on the amount of adhesive wear, strong correlations between thermoelectric current profiles and adhesive wear patterns could be deduced. An additional test series deals with the promising approach of influencing thermoelectric currents using external, controlled currents to reduce the amount of adhesive wear, thus offering new insights into the correlation between currents and wear.

\section{EXPERIMENTAL SETUP}

\section{Thermoelectric Property Measurement}

Without external influences, the velocity of charge carriers is homogeneous inside a conductive material. If one end is heated, the electron speed at this end raises and results in an average velocity vector pointing toward the cold end. A thermodiffusion is provoked until moving charge carriers develop an electric field that counteracts and finally stops it. The resulting electron displacement is the basis for the Seebeck effect. A material-specific constant, the Seebeck coefficient, determines the potential difference in relation to temperature. It describes the mobility and number of free charge carriers and mainly depends on the material composition and only subordinated to other influences such as the deformation degree. ${ }^{24}$

If two different conductors are connected at the heated end, the aforementioned thermoelectric voltages occur. In a short circuit, those voltages lead to measurable circular currents. The thermoelectric voltage $U_{A B}$ depends on the difference between the Seebeck coefficients of both materials $S_{A}$ and $S_{B}$ and is proportional to the temperature gradient: ${ }^{25}$

$$
\begin{aligned}
U_{A B}(T) & =\int_{T_{\text {ref }}}^{T_{\text {hot }}}\left(S_{B}(T)-S_{A}(T)\right) \mathrm{d} T \\
& =\left(S_{B}(T)-S_{A}(T)\right) *\left(T_{\text {hot }}-T_{\text {ref }}\right)[V]
\end{aligned}
$$


As the determination of thermoelectric properties or rather Seebeck coefficients is complex due to the manifold influence factors, the information in the scientific literature on most materials except semiconductors is insufficient and has to be determined experimentally. For this purpose, a special measuring apparatus is used, as shown in Fig. 1. Based on the integral measuring method, it utilizes a defined temperature gradient along a specimen. Therefore, one end of the sample is gradually heated up to $500^{\circ} \mathrm{C}\left(T_{\text {hot }}\right)$ while the other end is kept at the reference temperature of $0^{\circ} \mathrm{C}\left(T_{\text {ref }}\right)$ in an iced bath. A platinum wire serving as reference material connects both ends. The thermoelectric voltage $U_{A B}$ arises according to Eq. 1 . Since $U_{A B}$ amounts to only a few millivolts for the materials used, it needs to be amplified before recording. Figure 1 illustrates the measuring device with the sample in the middle. The prevailing temperatures at both sample ends are observed with high precision thermocouples to verify the temperature gradient and guarantee a high-quality measurement. These thermocouples are small sized to minimize the influences of heat transfer. The measurement of nickel-chromium and nickel samples, i.e., the two legs of a standardized type-K thermocouple, showed a maximum deviation of the measured thermoelectric voltage of $1.5 \%$.

\section{Blanking Tool Setup}

The utilized blanking tool has a four-pillar structure with a column diameter of $40 \mathrm{~mm}$, thus providing a very high degree of stiffness. Together with the modular design, it is suitable for different sheet materials and forming operations. As shown in Fig. 2, the punch, sheet metal, blankholder and die are electrically insulated from each other and the rest of the tool by zirconium oxide $\left(\mathrm{ZrO}_{2}\right)$ panels with a compression strength of $2300 \mathrm{MPa}$. This insulation suppresses interfering signals and guarantees a high degree of reproducibility. Whether these panels come in direct contact with the sheet metal or isolate metallic tool parts will not influence the measurement if there is no electrical contact with the rest of the tool and press. The punch force is measured by piezoelectric load cells with a measuring range up to $90 \mathrm{kN}$ in a force shunt configuration able to record tensile forces during the return stroke.

During all blanking experiments in this article, a $40-\mu \mathrm{m}$ die clearance, $1 \%$ of the sheet metal thickness and stroke rate of $601 / \mathrm{min}$, which corresponds to a punch impact speed of $50 \mathrm{~mm} / \mathrm{s}$, were chosen. Furthermore, an immersion depth of $4 \mathrm{~mm}$, circular punches with a diameter of $15 \mathrm{~mm}$ and cutting edge radii of $50 \mu \mathrm{m}$ were used. No lubricants were applied. All punches are ground to the same roughness to eliminate influences of surface conditions on adhesive wear development. ${ }^{26}$ The use of a highperformance mechanical stamping press with a

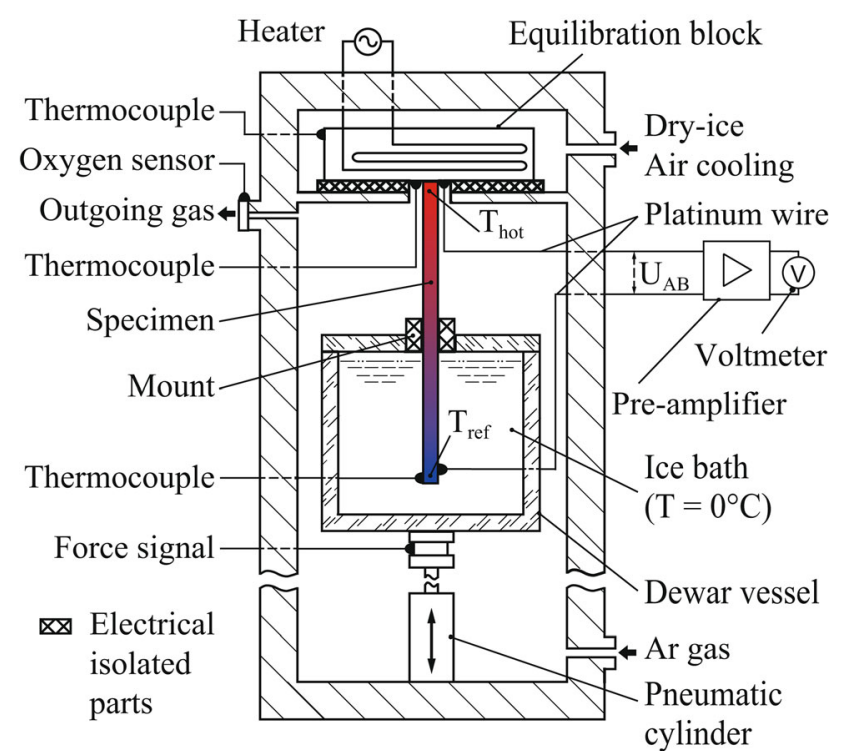

Fig. 1. Device for determining thermoelectric properties. Reprinted from Ref. 27 with the permission from Trans Tech Publication Ltd. Labeling changed from the original.

Source measure unit

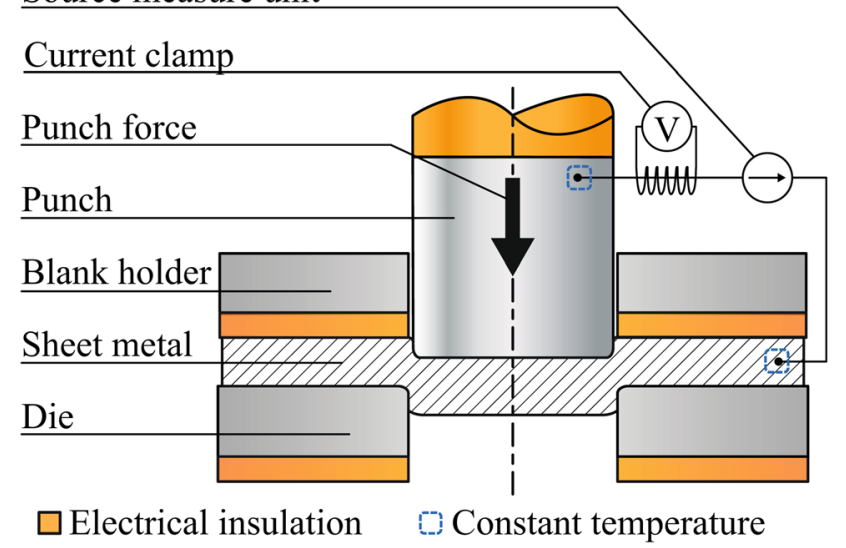

Fig. 2. Schematic diagram of the blanking tool.

maximum stamping force of $1600 \mathrm{kN}$ guarantees, in contrast to the hydraulic one used in former examinations, identical punch speed profiles for every experiment, independent of the process forces.

\section{Thermoelectric Current Measurement}

The zirconium oxide plates described above provide a specific electrical resistance of $10^{9} \Omega \mathrm{mm}$. Fiberglass washers insulate the tool screws with a specific electrical resistance of $10^{14} \Omega \mathrm{mm}$. Low noise cables connect the electric components, cutting punch and sheet metal to reduce currents induced by cable movement during the stroke. A low resistance, golden spring contact provides the electrical connection to the sheet metal. An amp clamp records occurring currents in a contact-free manner 
without retroactive effects. The connected amplifier converts the current signal to a voltage signal to guarantee a high-quality measurement. The force, current and punch travel values are recorded with a sample rate of $150 \mathrm{kHz}$. For countercurrent experiments, a source measure unit provides an external controlled current with an accuracy of $2.5 \mu \mathrm{A}$ and an adjusting time of about $1 \mathrm{~ms}$.

\section{Quantitative Adhesive Wear Measurement}

A confocal 3D laser microscope records the adhesive wear pattern in an optical spectrum. It uses a $0.95-\mathrm{mW}$ red semiconductor laser with a wavelength of $658 \mathrm{~nm}$ and a vertical accuracy of $0.7 \mu \mathrm{m}$. For wear quantity analysis, a worn area, having a width of $1 \mathrm{~mm}$ on the stamp's lateral surface, is recorded, and the adhesive wear volume is measured. Subsequently, the stamp roughness is subtracted to consider manufacturing disparities. The resulting wear coefficient (VQ) indicates the amount of adhesive wear as the height of the transferred material under the assumption of a homogeneous distribution.

\section{Materials Investigated}

The sheet metal used is the aluminum alloy EN AW 5083 (AlMg4.5Mn07) with a thickness of $4 \mathrm{~mm}$ and tensile strength of $270 \mathrm{MPa}$. The high-speed steel 1.3343 (X82WMoV65) with a hardness of $62 \mathrm{HRC}$ and Young's modulus of $224 \mathrm{GPa}$ as well as powder metallurgical cemented carbide CF-H40S with a hardness of 1400 HV10 and a Young's modulus of $551 \mathrm{GPa}$ serve as the tool material because of their frequent use in blanking operations. The austenitic stainless steel 1.4301 (X5CrNi18-10) with a hardness of 23 HRC, Young's modulus of $200 \mathrm{GPa}$ and good corrosion resistance is additionally used as a tool. The chemical composition of the used materials is shown in Table I. All three punch materials have an electrical resistance of around $10^{-4} \Omega / \mathrm{mm} ; 1.4301$ has a heat conductivity of $15 \mathrm{~W} /(\mathrm{m} \mathrm{K})$ compared with $22 \mathrm{~W} /(\mathrm{m} \mathrm{K})$ for 1.3343 and about $90 \mathrm{~W} /(\mathrm{m} \mathrm{K})$ for CF-H40S.

\section{PHENOMENA INFLUENCING ADHESIVE WEAR}

Essential to the emergence of adhesive wear is a close contact between the tool and sheet material. Since metal surfaces are not homogeneous, there are plenty of microscopic contact areas, some with an expansion of only a few atoms, e.g., roughness asperities or previous adhesive wear. ${ }^{28}$ In such sections, high surface pressures occur that impair the passivating oxide layer. Consequently, highly reactive metallic ions come in contact. ${ }^{2}$ This allows chemical and electrical phenomena to influence adhesive wear.

With the missing oxide layer as barrier, electrons can move freely between the contact partners and valence electrons are shared. This causes a strong chemical compound that exceeds the cohesive bonding strength in the sheet metal, and further punch movement tears out material parts, which accumulate at the punch. Due to an intense local contact, these adhesions serve as nucleation centers for further material transfer. ${ }^{28}$

In addition to the function of the free electrons for the chemical reaction, moving charge carriers influence these interrelations depending on direction and strength. If a directed electron flow, e.g., a thermoelectric current occurs, the small contact areas at the roughness asperities provoke a very high current density even in case of small current strengths. This entails two forces on the metal lattice elements. A strong force in electron flow direction occurs because of a transfer of kinetic energy through impact processes, similar to electromigration known from electronic components with extremely small wire cross sections. ${ }^{29,30}$ An opposing weak force is induced by the electric field. These forces result in a movement of metal ions contrary to the technical current direction. Since the difference between the Seebeck coefficients of two materials specifies the thermoelectric current direction, the choice of material will directly affect material transfer.

The current density also triggers the second effect, which is independent of the electron flow direction but highly sensitive to current strength. Induced plastic work to deform roughness asperities plus Joule heating from the current flow provokes temperatures that exceed the melting point of the sheet metal. The liquid metal resolidifies and adheres to the punch independent of the thermocurrent direction. ${ }^{1-33}$

Due to the interdependency of wear causing phenomena and current direction as well as strength, external currents can counteract or enhance the naturally occurring thermoelectricity and thus inhibit or intensify the transfer of sheet

Table I. Chemical composition of the materials investigated (in weight percentage)

\begin{tabular}{|c|c|c|c|c|c|c|c|c|c|c|c|c|}
\hline & $\mathbf{C}$ & $\mathbf{S i}$ & $\mathbf{C r}$ & Mo & $\mathbf{V}$ & $\mathbf{W}$ & $\mathbf{N i}$ & Mg & WC & Al & $\mathbf{F e}$ & Co \\
\hline 1.3343 & 0.8 & 0.4 & 4.2 & 8.4 & 2.2 & 5.6 & 0.3 & - & - & - & Balance & 0.7 \\
\hline 1.4301 & 0.3 & 0.4 & 13.8 & - & - & - & 1.3 & - & - & - & Balance & - \\
\hline CF-H40S & - & - & - & - & - & - & - & - & 88.0 & - & - & Balance \\
\hline EN AW 5083 & - & 0.1 & 0.1 & - & - & - & - & 5.8 & - & Balance & 0.1 & - \\
\hline
\end{tabular}


metal ions to the punch. With the goal of the reduction of adhesive wear pattern, the controlled current consequently has to be adjusted with respect to the materials in contact. Given the wrong direction, the external current will raise the thermocurrent strength and cause more impact processes. If the controlled current strength is too high, melting processes will prevail. Both factors have a negative influence on the adhesive wear pattern.

\section{RESULTS}

This investigation focuses on the thermoelectric currents caused by the Seebeck effect, their influence on adhesive wear development and the impact of an external, controlled current on wear pattern. The basis is the determination of the thermoelectric properties. Given both this knowledge and the aforementioned measurement and tool setup, twofold blanking test series were performed: first, a variation of the punch material between 1.3343, 1.4301 and CF-H40S; second, with punches made of 1.3343 and an external, controlled countercurrent. Every experiment series contained nine strokes; after that the adhesive wear pattern was qualified regarding the amount and local dispersion. The force and current profiles shown, with the exception of the external current strength, are averages of all nine strokes.

\section{Seebeck Coefficients}

The temperature range for the determination of the thermoelectric properties was $0^{\circ} \mathrm{C}$ to $500^{\circ} \mathrm{C}$ in which the three investigated tool materials showed varying behaviors. Figure 3 illustrates the difference between their Seebeck coefficients and the aluminum alloy EN AW 5083. While the high-speed steel 1.3343 shows a positive Seebeck coefficient, the one of cemented carbide is negative, which causes different current flow directions. The difference of the Seebeck coefficient profile of EN AW 5083 and

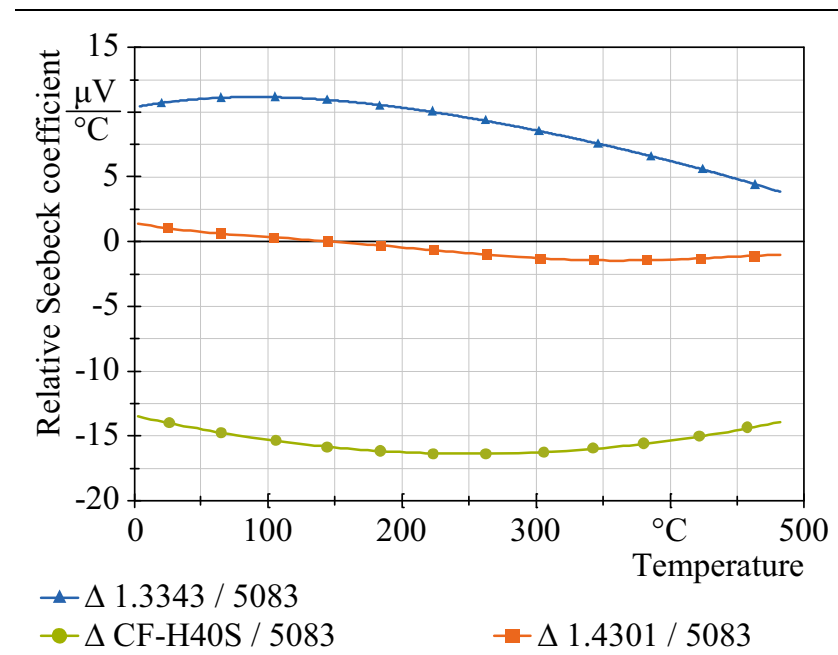

Fig. 3. Relative Seebeck coefficients for 1.3343, CF-H40S and 1.4301 referred to 5083 .
1.4301 is very small, especially in the temperature range of the estimated process temperature between $50^{\circ} \mathrm{C}$ and $100^{\circ} \mathrm{C}$. Consequently, only low thermoelectric current flows. The highest difference and thus thermocurrents arise between CF-H40S and aluminum. The resulting thermocurrent will be lower and contrarily directed, when using 1.3343 .

\section{Punch Forces}

Figure 4(a) illustrates the averaged punch force profiles of the four experiment setups, with negative punch travel values representing movement toward the bottom dead center. A blanking process is dividable into five sections with characteristic curve shapes, as illustrated in Fig. 4 (I V). First, a linear increase starting at $-8 \mathrm{~mm}$ before the bottom dead center primarily correlates with the elastic deformation of the sheet metal and the tool (Section I). In the second part of the process (II), starting at about $7.6 \mathrm{~mm}$, the initiation of plastic deformation takes place, the clean cut is formed, and the maximum force of $34 \mathrm{kN}$ is reached. Afterwards, microscopic cracks expatiate and the force decreases. The third section (III) starts at $5.4 \mathrm{~mm}$. Crack expansion leads to a total material severance and fractured cutting surface. Caused by the low die clearance, also a secondary clean cut arises. At $4.0 \mathrm{~mm}$, the punch front surface reaches the sheet metal thickness and the slug is pushed out through the die (section IV). Wedged slugs from the prior strokes lead to an increase of the force profile. Since this rise does not represent a forming force, no work dissipates and no temperature increase in the contact zone between the punch and sheet metal occurs. Therefore, the thermoelectric currents and thus adhesive wear pattern are not affected. Consequently, the force increase is neglected, as shown in Fig. 4(a) as dashed lines. The bottom dead center ends this fourth part, where the return stroke (section V) begins.

The Young's modulus of EN AW 5083 determines the shape of all four graphs in the first section. After that, the graphs flatten; the strongest pronounced the 1.3343 countercurrent punch, which attains the maximum force later than the other three punches and exhibits, beside the stainless steel one, the lowest force maximum. Subsequently, stainless steel and high-speed steel with a countercurrent remain on a high level, while cemented carbide and the high-speed steel forces that are not influenced decrease. The profile of CF-H40S goes down almost constantly until the end of the third section, whereas the steel punches demonstrate a varying behavior. Those three force profiles show a strong curvature change while the force is declining, with the strongest pronounced for 1.4301.

After material disruption, frictional forces preponderate over the plastic work. The neglected increase and the assumption of a constant pushout force for the prior slug in the fourth phase show 

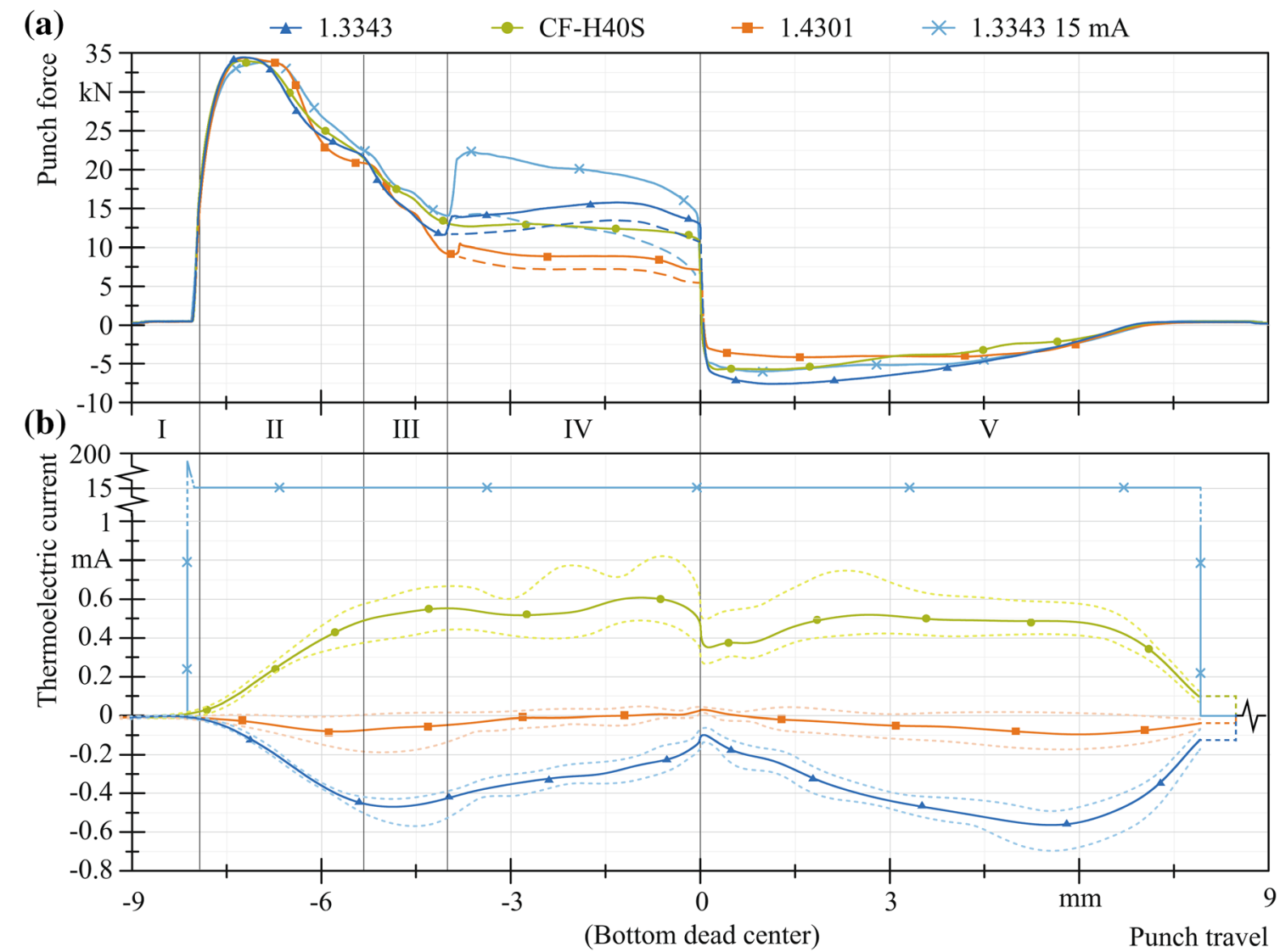

Fig. 4. (a) Punch forces and (b) thermoelectric currents as well as an exemplary external, controlled current of $15 \mathrm{~mA}$ over punch travel for 1.3343, CF-H40S and 1.4301.

that the forces for CF-H40S and 1.3343 with countercurrent are comparable; both reach $>12$ $\mathrm{kN}$. A second slug from the previous stroke causes the high force increase of 1.3343 with countercurrent. As mentioned above, this does not affect the thermoelectricity. The force level of the not externally influenced 1.3343 is slightly smaller but increases afterwards and exceeds the other two. That of 1.4301 , at around $8 \mathrm{kN}$, is significantly lower compared with the other punches.

The punch movement stops at the bottom dead center, and the return stroke begins, whereby the force levels are $3 \mathrm{kN}$ for $1.4301,5.5 \mathrm{kN}$ for CF-H40S as well as 1.3343 with countercurrent and $6.3 \mathrm{kN}$ for 1.3343. Afterwards, the force profiles of the stainless steel and cemented carbide cross each other before the punch and sheet metal lose contact.

\section{Thermoelectric Currents}

Figure 4(b) shows the thermoelectric current profiles against the punch travel. Positive current values represent an electron flow from the punch to sheet metal, negative ones the contrary direction. Beyond the averaged current profiles, maximum and minimum values are illustrated as dashed lines. The thermocurrent graphs of the three materials exhibit substantially different behaviors and can also be divided into the five aforementioned sections. For the cemented carbide, electrons shift from the punch to sheet metal; 1.3343 and the stainless steel trigger the opposed direction, whereby the maximum thermocurrent of 1.4301 is very low and only reaches values $<0.1 \mathrm{~mA}$. In the first phase of blanking (I), almost no current flow is measurable because no plastic work is conducted and no temperature increases take place. Actually, very little cooling occurs because of the JouleThomson effect applied to metals. ${ }^{34}$ In the second section (II), a strong rise of the current strength with a curvature change at the force maximum occurs. This can be traced back to a temperature raise until the end of the clean-cut formation. During the development of the fractured surface and secondary clean cut (section III), the current strength and thus the temperature are nearly constant. In the push-out phase (IV), the current strength of CF-H40S is constant while that of 1.3343 slightly declines. At the bottom dead center, a sharp decrease of the current strength occurs. After that (section V), a new increase to the magnitude of the maximum currents of the first three sections happens. In the following, the declining 
force toward the end of the stroke causes the current to descend. After the punch loses contact with the sheet metal, the short circuit breaks, and no more current can be measured.

\section{External Countercurrent}

An external generated current varying in the range from $1 \mathrm{~mA}$ to $20 \mathrm{~mA}$ in different directions is the basis for the countercurrent experiments. Figure 4(b) illustrates an exemplary profile of the applied current. A value set to $15 \mathrm{~mA}$ results in the lowest amount of wear. The external countercurrent reaches the defined constant value after the adjusting time, which starts when the sheet metal and punch come in contact, thus resulting in electric contact. To keep the influence of the initial overshoot as low as possible, the adjusting time has to be short enough to gain the exact target value before plastic deformation begins. For the experiments with a countercurrent strength of $15 \mathrm{~mA}$, the adjusting time is about $1.3 \mathrm{~ms}$ where the force reaches $4.92 \mathrm{kN}$, which corresponds to an immersion depth of $0.08 \mathrm{~mm}$ compared with the start of plastic deformation at $0.4 \mathrm{~mm}$. The highest overshoot of the currents of up to $193 \mathrm{~mA}$ influences the electric conditions for $<80 \mu \mathrm{s}$.

\section{DISCUSSION}

\section{Force Profiles}

Three effects cause differing maximum forces and profiles in the second section. First, the friction conditions vary measurably through the die roll at the stamping grid and slug. The die roll at the slug for cemented carbide is $20 \%$ bigger than for the other tool materials, which means lower frictional forces between the stamping grid and the punch shell surface. Lower friction causes an earlier crack initiation due to smaller compression stress in the shearing zone, which can be seen in a lower cleancut proportion. The second effect influences force after the maximum because of different punch compressions in the movement direction. This is caused by different Young's moduli of the three punch materials. A numerical calculation for the 1.4301 punch displays a force-induced radial widening of $7 \mu \mathrm{m}$ in the area of the cutting edge. The resulting smaller die clearance enhances the hydrostatic compression stress and thus delays crack initiation. ${ }^{35}$ This effect is amplified by adhesions close to the cutting edge with an expansion of about $5 \mu \mathrm{m}$ for 1.4301 . The clean-cut proportion substantiates the numerical results, as it amounts to $75 \%$ for stainless steel, 69\% for high-speed steel and $60 \%$ for cemented carbide. Further finite element simulations show expansions of $5 \mu \mathrm{m}$ for the not externally influenced 1.3343 punch and a small widening of $2 \mu \mathrm{m}$ for the CF-H40S punch. Both have no adhesions close to the cutting edge. The 1.3343 punch with countercurrent shows a radial widening of $5 \mu \mathrm{m}$ and additionally adhesive wear at the cutting edge with a height of $4 \mu \mathrm{m}$, which shifts the force profile in the area of the maximum force. Numerical stress calculation and laser microscopic imaging were able to disprove plastic deformation of the active elements, in particular the punch made of stainless steel.

The decreasing force in section(III) entails a reduction of the before-mentioned punch expansion for stainless and high-speed steel. The consequential lower frictional forces between the punch shell surface and clean cut in the stamping grid decrease the forming force profile.

As mentioned before, the force increase in section (IV), caused by the clamping slugs from previous strokes, can be neglected. Frictional forces prevail in the fourth and fifth sections of the profile (IV and V). The lowest amount of adhesive wear on the 1.4301 punch shell surface minimizes frictional forces between the punch and stamping grid and causes the force to be lower than the other three punches. Due to the homogeneous wear pattern on the stainless steel and the 1.3343 countercurrent punches, in section (V) both force profiles remain constant until the overlap of the punch and sheet metal and thus the force declines. Therefore, the differing force levels of the four punches correspond to the quantity of adhesive wear. A higher number of adhesions leads to a higher friction force because of a rougher surface between the punch and stamping grid. Consequently, the punch force of the not influenced 1.3343 punch shows the highest level; cemented carbide is significantly lower and the influenced high-speed steel is in between.

\section{Current Profiles}

The similar Seebeck coefficients of 1.4301 and EN AW 5083 cause a very low current strength in the stainless-steel experiments. However, two effects cause a measurable current even with these punches. First, the Seebeck coefficients are not exactly equal in the relevant temperature range. The second reason is the influence of adhesive wear, even though the quantity is low. In the first stroke, with no adhesions, the thermocurrent strength is approximately zero.

Regarding the other steels, heat transfer cannot equalize the heat from dissipating plastic work. This causes the strong current rise in the second phase of blanking (II). The declining force and thereby reduced heating from dissipating plastic work, friction and a decreasing forming zone in balance with heat equalization explain the constant current of CF-H40S during the third section (III). The profile of 1.3343 shows a different behavior before the bottom dead center. This can be traced back to the differing contact area between the punch and sheet metal caused by the varying clean-cut proportion and thus a differing area where plastic work from shearing off adhesive wear is induced. 
Stainless steel with a clean-cut proportion of $69 \%$ has, compared with cemented carbide with $60 \%$, a wider contact area and thus lower maximum temperatures and higher heat transfer.

Near the bottom dead center while the punch moves slowly or stands still, heat transfer effects trigger the sharp decrease. Cemented carbide shows a bigger reduction due to higher heat conductivity. A clamping effect that increases friction and plastic work for equalization of roughness asperities entails a strong repeated increase of the temperatures and thus the thermocurrent strength during the return stroke. The ascent for the 1.3343 punch profile is bigger because of the higher force level and the lower heat conductivity.

\section{Correlation Between Thermoelectricity and Adhesive Wear Development}

While Fig. 5 illustrates the distribution of adhesions on the lateral surface of the respective punch, Fig. 6 shows the amount of adhesive wear. For the three punch materials and the countercurrent experiment, Table II additionally indicates the relative Seebeck coefficient at the approximate maximum process temperature of $75^{\circ} \mathrm{C}$ and the appendant maximum current strength. All four experiments show significant variations in the adhesive wear pattern. CF-H40S generates only $56 \%$ of the amount of adhesions compared with 1.3343 although the maximum current strength is, according to amount, higher for cemented carbide.
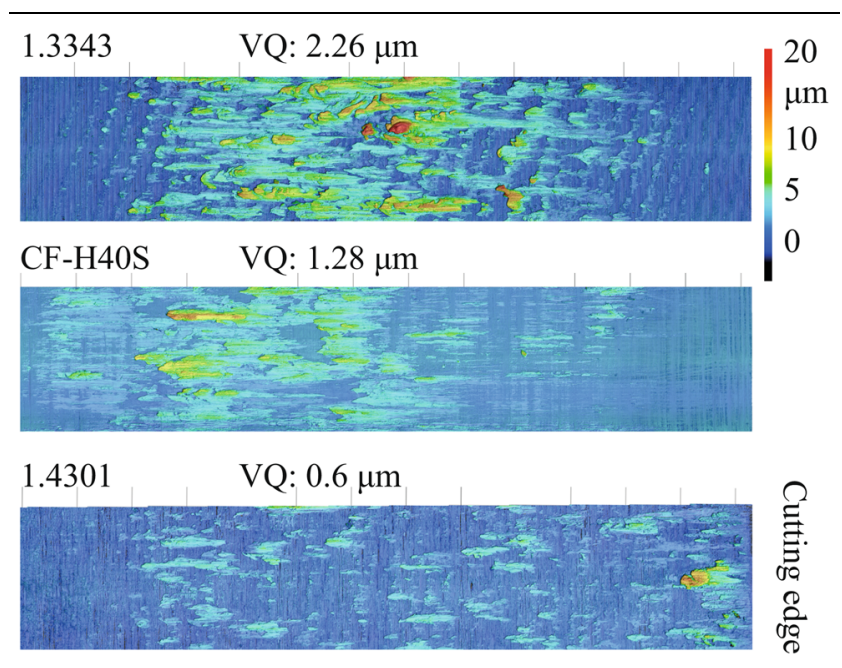

$1.334315 \mathrm{~mA} \quad \mathrm{VQ}: 1.6 \mu \mathrm{m}$

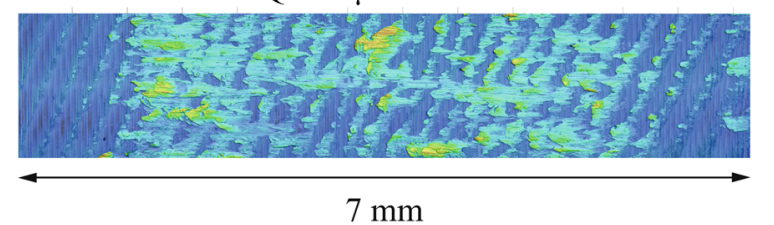

Fig. 5. Laser microscopic pictures of the adhesive wear distribution on the four cutting punches shell surfaces.
The opposing electron flow directions show a strong impact on the amount of adhesive wear. While an electron flow from the sheet metal to punch enhances material transfer, like in the case of 1.3343 , the opposite direction counteracts adhesive wear formation, as with CF-H40S. This can be attributed to the aforementioned collision processes between electrons and the metal lattice;

1.4301 shows the lowest amount of adhesions with only $27 \%$ of 1.3343 and $47 \%$ of CF-H40S. Considering the thermoelectric profile in Fig. 4(b), the low thermocurrent strength is obvious. Consequently, melting and electromigration only have a minor influence. Nevertheless, chemical bonding due to the exchange of valence electrons takes place even without current flow. Consequently, little adhesive wear develops even with similar Seebeck coefficients of punch and sheet metal; 1.3343 with a countercurrent of $15 \mathrm{~mA}$ has a wear coefficient of $1.6 \mu \mathrm{m}$, which corresponds to $71 \%$ of the amount of the not influenced high-speed steel punch surface and $125 \%$ of cemented carbide. The external current inverts the thermoelectric current flow with a constant strength of $15 \mathrm{~mA}$. It stops the effect of electromigration and reduces melting processes because of a lower maximum current. Therefore, influencing naturally occurring thermoelectricity with external currents and thus adhesive wear development is possible.

Regarding the 1.3343 punch surface, no adhesions at the cutting edge occur. After $0.5 \mathrm{~mm}$ a slight rise takes place until reaching the maximum quantity from $3 \mathrm{~mm}$ up to $4.5 \mathrm{~mm}$. After that, the number of adhesions declines. A correlation with the thermoelectrical current profile is evident as it increases at $0.5 \mathrm{~mm}$ after contact of the punch and sheet metal and reaches its maximum at $5 \mathrm{~mm}$ before the bottom dead center. CF-H40S shows an analogical behavior: no adhesions at the cutting edge, a slight rise and a maximum at the point where the maximum current is reached. The adhesive wear pattern of 1.4301 is completely different. An instant but less pronounced maximum close to the cutting edge and, after that, a low and homogeneous distribution occur. The elastic punch deformation in combination with melting processes induced by interaction of the heat from dissipating plastic work and the low heat conductivity of 1.4301 cause the material concentration at the edge. Chemical bonding due to valence electron exchange provokes the homogeneous wear area on the residual punch shell surface.

The amount of adhesive wear on the externally influenced punches varies with current strength and direction. While the wear coefficient declines with higher current strength, it reaches a minimum at $15 \mathrm{~mA}$ and subsequently rises again. The external current counteracts the naturally occurring thermoelectricity and reduces the current flow in the contact area between the punch and sheet metal. This lowers the kinetic energy transferred 


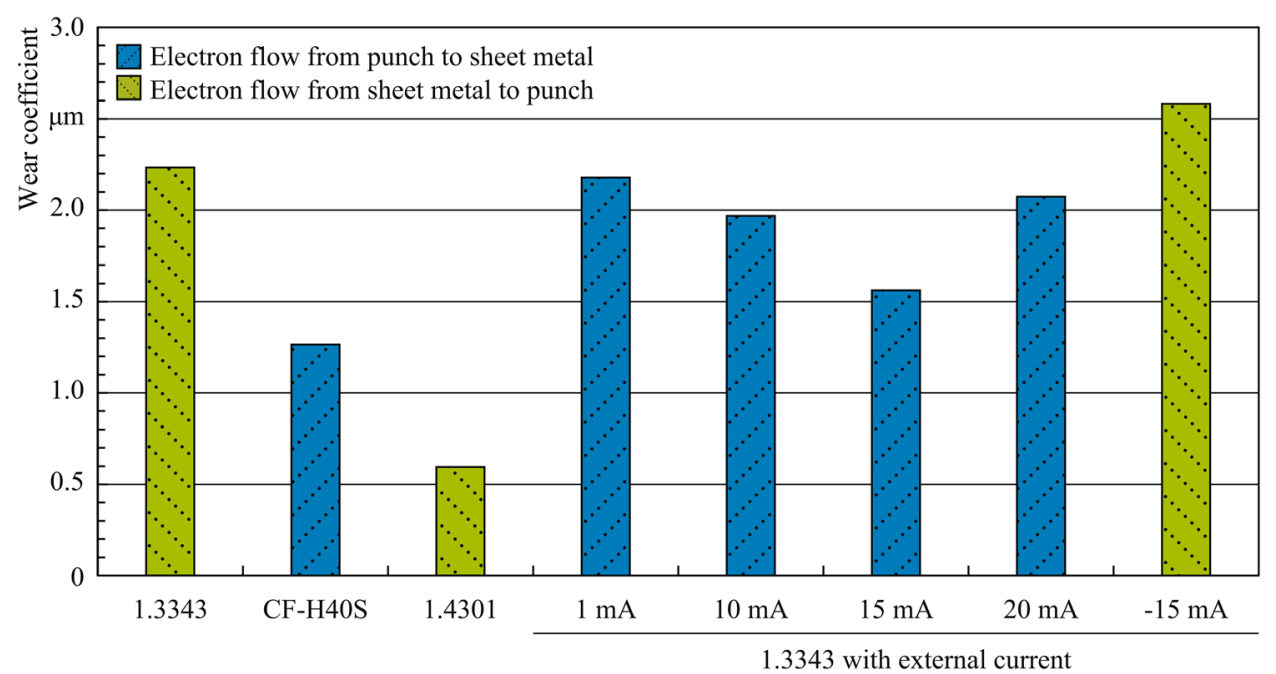

Fig. 6. Wear coefficients for the three punch materials and for external currents with different strengths and direction.

Table II. Wear coefficient, maximum thermocurrent and seebeck coefficients of the three punch materials; $1.334315 \mathrm{~mA}$ represents the countercurrent experiment

\begin{tabular}{|c|c|c|c|}
\hline Punch material & Wear coefficient (VQ) $[\mu \mathrm{m}]$ & Maximum current $[\mathrm{mA}]$ & Relative Seebeck coefficient $\left[\mu \mathrm{V} /{ }^{\circ} \mathrm{C}\right]$ \\
\hline 1.3343 & 2.26 & -0.47 & 11.2 \\
\hline CF-H40S & 1.28 & 0.59 & -15.1 \\
\hline 1.4301 & 0.6 & -0.07 & 0.3 \\
\hline $1.334315 \mathrm{~mA}$ & 1.6 & 15.00 & 11.2 \\
\hline
\end{tabular}

from electrons to metal ions and Joule heating. If the countercurrent strength becomes excessive, melting processes prevail and the amount of adhesive wear grows. If the external current direction matches the thermocurrent direction, both processes are strengthened and the number of adhesions exceeds the 1.3343 punch without external current.

The wear pattern on the externally influenced high-speed steel punch also shows an accumulation at the cutting edge, but the basic reason is different. In this case, the external generated current is much higher than the naturally arising thermocurrents. Together with the small contact area between the punch and sheet metal before the beginning of the plastic deformation, sheet material in the microcontacts melts because of Joule heating. After that, in contrast to the not influenced high-speed steel punch, few adhesions occur because of melting effects caused by an overcompensation of the low thermocurrent. Subsequently, the number of adhesions increases and reaches a significantly lower pronounced maximum. In this area, the naturally occurring thermocurrent shows the highest strength, and thus the countercurrent compensation takes effect and leads to a reduction of wear. Overall, the adhesive wear pattern with external current is much more homogeneous compared with the 1.3343 punch without those currents.

\section{CONCLUSION}

In this article, fundamental correlations among material choice, thermoelectric currents and adhesive wear were derived. Therefore, the Seebeck coefficient of different materials was first determined. In addition, a special blanking tool setup is presented that allows an instantaneous and simultaneous measurement of process forces and thermoelectric currents during blanking. It also provides the ability to influence thermoelectricity by external, controlled currents. With this setup, several blanking experiments were conducted using aluminum sheet metal EN AW 5083. The occurring adhesive wear at the shell surface of the punch was analyzed after nine strokes. Stainless steel 1.4301, high-speed steel 1.3343 and cemented carbide CFH40S were chosen as sheet material. Their varying thermoelectric properties, represented by the Seebeck coefficient, lead to different thermoelectric current strengths and directions during blanking. While the algebraic sign of the difference between the Seebeck coefficients of punch and sheet material defines the current direction, the current strength depends on not only the temperature but also on the absolute value of the difference. Although the thermoelectric current profiles differ in amount and direction, they show a characteristic course with respect to the five phases of blanking. Varying material behaviors and properties such as the 
elastic deformation of the punch and the materialspecific heat conductivity additionally influence the thermoelectric current profile.

Regarding adhesive wear, an interrelation among the quantity, distribution and the measured thermoelectric current profiles can be seen. The results obtained confirm the findings of Tröber et al. for a new parameter setup and another tool material. ${ }^{22}$ Furthermore, a detailed analysis of the wear pattern with a laser confocal microscope shows the correlation between the thermocurrent curve and the development of adhesive wear. Therefore, knowledge about the fundamental wear causing interactions could be improved. In general, the formation of adhesion can be traced back to chemical bonding because of an exchange of electrons as well as melting caused by a superposition of Joule and frictional heating as well as a temperature increase based on dissipating plastic work. The amount of adhesive wear increases with higher currents but the current direction also has a significant influence. Collision processes between electrons and the metal lattice cause a strengthening of the material transfer in case of an electron flow direction from the sheet metal to punch and a contrary effect in the opposite direction. In case of similar Seebeck coefficients of the sheet metal and punch, no thermoelectric current occurs and the adhesive wear is minimized. Furthermore, regarding adhesive wear development, for every material combination an ideal countercurrent strength exists, depending on the thermoelectric properties and boundary conditions. Thus, counteracting the naturally occurring thermocurrents with an external, controlled current offers the possibility to change the adhesive wear pattern regarding the amount and distribution. Consequently, influencing thermoelectric effects by a material choice according to its Seebeck coefficient or by external currents is a promising approach to reduce adhesive wear and optimize the blanking process for future applications.

\section{ACKNOWLEDGEMENTS}

Open Access funding provided by Projekt DEAL. This work was supported by the German Research Foundation (DFG) (Grant No. VO 1487/6-3).

\section{OPEN ACCESS}

This article is licensed under a Creative Commons Attribution 4.0 International License, which permits use, sharing, adaptation, distribution and reproduction in any medium or format, as long as you give appropriate credit to the original author(s) and the source, provide a link to the Creative Commons licence, and indicate if changes were made. The images or other third party material in this article are included in the article's Creative
Commons licence, unless indicated otherwise in a credit line to the material. If material is not included in the article's Creative Commons licence and your intended use is not permitted by statutory regulation or exceeds the permitted use, you will need to obtain permission directly from the copyright holder. To view a copy of this licence, visit http://creativecommons.org/licenses/by/4.0/.

\section{REFERENCES}

1. R.S. Long, E. Boettcher, and D. Crawford, JOM 12, 2635 (2017).

2. H. Czichos, Meccanica 6, 605 (2001).

3. P. Groche, G. Nitzsche, and A. Elsen, CIRP Ann. 1, 295 (2008).

4. P. Groche and G. Nitzsche, J. Mater. Process. Technol. 1-3, 314 (2007).

5. T. Sasada, S. Norose, and H. Mishina, J. Lubr. Technol. 2, 195 (1981)

6. F. Sergey and J. Golovashchenko, Mater. Eng. Perform. 2, 213 (2007).

7. R. Hambli, Int. J. Mach. Tools Manuf. 12, 1815 (2001).

8. F. Vollertsen and F. Schmidt, Int. J. Precis. Eng. Manuf.Green Technol. 1, 59 (2014).

9. G.W. Stachowiak and A.W. Batchelor, Engineering Tribology, 4th ed. (Oxford: Butterworth-Heinemann, 2014).

10. R.D. Barnard and V. Cannella, Phys. Today 9, 52 (1974).

11. P. Demmel, T. Kopp, R. Golle, and W. Volk, Steel Res. Int., 1, 291 (2012).

12. P. Demmel, H. Hoffmann, R. Golle, C. Intra, and W. Volk, CIRP Ann. 1, 249 (2015).

13. T. Alan, Zehnder. Mech. Res. Commun. 1, 23 (1991).

14. J. Schulz and W. Holweger, Wechselwirkung von Additiven mit Metalloberflächen (Renningen-Malmsheim: Expert Verlag, 2010).

15. P. Tröber, M. Welm, H.A. Weiss, P. Demmel, R. Golle, and W. Volk, MATEC Web Conf. (2018). https://doi.org/10.1051/ matecconf/201819014004.

16. A.A. Awakow and A. Ritschkin, Masch. Fert. UdSSR 68, 19 (1976).

17. H. Opitz and W. König, Advances in Machine Tool Design and Research 1967 (Amsterdam: Elsevier, 1968), pp. 173190.

18. V.A. Bobrovskij, Masch. Fert. UdSSR 76, 267 (1976).

19. J. Ellis and G. Barrow, CIRP Ann. 17, 39 (1969).

20. H.S. Shan and P.C. Pandey, Wear 2, 167 (1975).

21. K. Uehara, M. Sakurai, and T. Ikeda, CIRP Ann. 1, 75 (1992).

22. P. Tröber, P. Demmel, H. Hoffmann, R. Golle, and W. Volk, CIRP Ann. 1, 293 (2017).

23. P. Tröber, H.A. Weiss, T. Kopp, R. Golle, and W. Volk, Int. J. Mach. Tools Manuf. 118, 91 (2017).

24. P. Demmel, A. Pazureck, R. Golle, W. Volk, and H. Hoffmann, J. Electron. Mater. 7, 2371 (2013).

25. R. Pelster, I. Hüttl, and R. Pieper, PhyDid 1, 10 (2005).

26. D.H. Buckley, Surface Effects in Adhesion, Friction, Wear, and Lubrication (Amsterdam: Elsevier, 2010).

27. P. Demmel, P. Tröber, T. Kopp, R. Golle, W. Volk, and H. Hoffmann, Mater. Sci. Forum 1 (2013).

28. P. Groche and F. Resch, Materialwiss. Werkstofftech. 8, 813 (2015).

29. S.-H. Lee and C.-M. Chen, J. Electron. Mater. 9, 1943 (2011).

30. A.S. Budiman, C.S. Hau-Riege, W.C. Baek, C. Lor, A. Huang, H.S. Kim, G. Neubauer, J. Pak, P.R. Besser, and D. Nix, J. Electron. Mater. 11, 2483 (2010).

31. A. Hase and H. Mishina, Tribol. Int. 127, 372 (2018).

32. A. Ber and M. Goldblatt, CIRP Ann. 38, 69 (1989). 
33. L. Lind, P. Peetsalu, P. Põdra, E. Adoberg, R. Veinthal, and P. Kulu, Description of punch wear mechanism during fine blanking process, in 7th International DAAAM Baltic Conference (2010).

34. S. Vitzthum, C. Hartmann, M. Eder, and W. Volk, Procedia Manuf. 29, 490 (2019).
35. H. Hoffmann, R. Neugebauer, and G. Spur, Handbuch Umformen (Munich: CARL HANSER Verlag GMBH, 2012).

Publisher's Note Springer Nature remains neutral with regard to jurisdictional claims in published maps and institutional affiliations. 